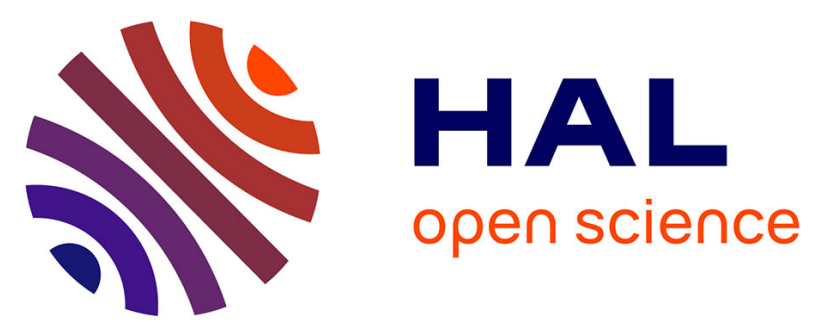

\title{
Planar Hall sensor for quantitative measurement of pipe wall thickness reduction based on the magnetic flux density method
}

Hong-Quang Pham, Trung-Kien Nguyen, Quang-Ngan Pham, Van-Sy Le, Minh-Hung Vu, Thi-Thuy Truong, Van-Tai Nguyen, Azzedine Bousseksou, Sidina Wane, Ferial Terki, et al.

\section{To cite this version:}

Hong-Quang Pham, Trung-Kien Nguyen, Quang-Ngan Pham, Van-Sy Le, Minh-Hung Vu, et al.. Planar Hall sensor for quantitative measurement of pipe wall thickness reduction based on the magnetic flux density method. Measurement - Journal of the International Measurement Confederation (IMEKO), 2021, 182, pp.109782. 10.1016/j.measurement.2021.109782 . hal-03285765

\section{HAL Id: hal-03285765 https://hal.science/hal-03285765}

Submitted on 13 Jul 2021

HAL is a multi-disciplinary open access archive for the deposit and dissemination of scientific research documents, whether they are published or not. The documents may come from teaching and research institutions in France or abroad, or from public or private research centers.
L'archive ouverte pluridisciplinaire HAL, est destinée au dépôt et à la diffusion de documents scientifiques de niveau recherche, publiés ou non, émanant des établissements d'enseignement et de recherche français ou étrangers, des laboratoires publics ou privés. 


\title{
Planar Hall sensor for quantitative measurement of pipe wall thickness reduction based on the magnetic flux density method
}

\author{
Hong-Quang Pham ${ }^{1, *}$, Trung-Kien Nguyen ${ }^{2,3}$, Quang-Ngan Pham ${ }^{4}$, Van-Sy Le ${ }^{1}$, Minh-Hung \\ $\mathrm{Vu}^{1}$, Thi-Thuy Truong ${ }^{1}$, Van-Tai Nguyen ${ }^{2}$, Azzedine Bousseksou ${ }^{5}$, Sidina Wane $^{6}$, Ferial Terki ${ }^{2, *}$, \\ Quang-Hung Tran ${ }^{6, *}$ \\ ${ }^{1}$ PetroVietnam University, 762 Cach Mang Thang Tam Road, Baria City 79000, Vietnam \\ 2 PhyMedExp, UMR CNRS 9214 - Inserm U1046, CHU Montpellier, Université de Montpellier, \\ Montpellier 34095, France \\ ${ }^{3}$ Institute of Environmental Technology, Vietnam Academy of Science and Technology, 18 \\ Hoang Quoc Viet, Cau Giay, Hanoi 10000, Vietnam \\ ${ }^{4}$ Institute of Materials Science, Vietnam Academy of Science and Technology, 18 Hoang Quoc \\ Viet, Cau Giay, Hanoi 10000, Vietnam \\ ${ }^{5}$ Laboratoire de Chimie de Coordination UPR 8241, CNRS, 31007 Toulouse Cedex 4, France \\ ${ }^{6}$ eV-Technologies, Caen 14000, France \\ *E-mail: quangph@pvu.edu.vn; ferial.terki@umontpellier.fr; quang-hung.tran@ev- \\ technologies.com
}

\begin{abstract}
Quantitatively monitoring the thickness reduction of a pipe by using magnetic flux density method remains a major challenge due to the small and gradual change in magnetic flux density over a long distance along a pipe. This signal change is hindered in the background signal of a detector that has a high temperature dependence and unstable background signal.

In this paper, we verify the effectiveness of magnetic flux density method in quantitatively monitoring thickness reduction through simulation and experimental verification. Experimental results show that the proportion of magnetic signal change is $1.4 \mathrm{mT}$ per $10 \%$ wall loss.

To support this quantitative approach, we optimise an original ultra-high-sensitivity planar Hall sensor with high thermal stability and stable background signal versus time for measurement of the magnetic field over a wide range from 0 to $50 \mathrm{mT}$. The quantitative measurements were validated on test pipes with varying thickness steps of $0.5 \mathrm{~mm}$.
\end{abstract}

Keywords: magnetic flux density, thickness monitoring, planar Hall sensor, ultra-highsensitivity, high stability. 


\section{Introduction}

The wall thickness of a pipe can decrease during the production process or during in-service life. In the production process, metal loss may occur in the raw material or during pipe manufacturing. During in-service use, metal loss occurs due to corrosion and erosion. All types of metal losses may result in catastrophic accidents. Therefore, the condition of pipes needs to be examined before the employment of new pipes and at regular intervals throughout the use of the pipe system. Several electromagnetic techniques for pipe wall loss inspection have been introduced, such as electromagnetic acoustic transducer [1-2], remote-field eddy current [3-4], total magnetic flux [5-6], magnetic flux leakage [7-8] and magnetic flux density [9].

Wall thickness monitoring is one of the important criteria for examination of a pipe system, but small thickness variations over a long distance along a pipe make this process a major challenge. Concerning electromagnetic techniques for inspection of pipe wall loss, R. K. Stanley introduced a total flux method to measure the ferromagnetic cross section $[5,6]$. The method is based on the measurement of the total flux that passes an encircling detector coil, including flux in air and in pipe wall. Yunwei Zhang et al. [7] and Buddhi Wijerathna et al. [8] proposed a method for measuring the wall thickness of gas pipes by using electromagnetic flux leakage (MFL) technique. This method is somewhat like a pipeline inspection gauge system. In our opinion, the MFL method is more suitable for detecting defects that exhibit an abrupt change in the magnetic permeability of the pipe wall or an abrupt discontinuity of the cross-sectional area in the pipe than for measuring the thickness variation over a long distance with a metre scale.

For pipes with small, straight and disjointed characteristics, such as tubing pipes and drill pipes, the magnetic flux density method is the most suitable for monitoring and quickly identifying small changes in thickness along the length of a pipe body for other subsequent thickness 
measurement methods. The principle of the magnetic flux density measurement method can be

2 summarised as follows:

3 A coil formed by a number of current-carrying loops produces a magnetic field along the

4 longitudinal axis. When a ferromagnetic pipe is placed in the coil, flux lines within the coil tend

5 to travel in the ferromagnetic material, thereby reducing the magnetic flux density in the air

6 surrounding the pipe. Therefore, a pipe with thin walls will cause a small reduction of flux

7 density in air and vice versa. Noting that the variation of the flux density occurs for any thickness

8 change in the cross section area of the pipe, regardless the thinning or thickening of the pipe and

9 the location of defects at the inner or the outer surface. Variations in wall thickness can be

10 monitored by the change in the magnetic flux density with the use of a magnetic sensor. Thus,

11 the method is not an MFL method and does not measure wall thickness but rather the change in

12 wall thickness.

13 Although magnetic flux density measurement is a fast and effective method for monitoring the

14 thickness change in a pipe, few works have been published on this technique. To quantify the

15 wall loss of a pipe that undergoes gradual wall reduction, the major challenge of the flux density

16 method lies in the need of a magnetic sensor having (i) high sensitivity, low noise to measure

17 small change of wall thickness; (ii) wide field range for measurement in a high magnetic flux

18 density environment; and (iii) high temperature stability for stable and accurate measurement

19 during long time. In the field of magnetic sensors, high-sensitivity, low-noise sensors limit the

20 measurement in small magnetic fields range [10].

21 Among the few relevant reports, William Walters et al. developed a flux density measurement 22 system using a non-contact inspection ring composed of a series of conventional Hall sensors, 
which can measure high fields [6]. However, due to high noise in the measurement of magnetic

2 field and poor stability with temperature variation and time, the Hall sensor system deployed in

3 this referenced work is effective for qualitative evaluation of thickness variation only but not for

4 quantitative evaluation. Consequently, quantitative measurement of thickness change in a pipe

5 by using magnetic flux density method continues to be a challenge.

6 In this paper, we introduce an original approach to the quantitative measurement of pipe wall

7 thickness reduction by using the flux density method. The quantitative approach is achieved in

8 the sense of how much the magnetic flux density changes with respect to a given thickness

9 change of a pipe wall. To enable this quantitative measurement of a less abrupt signal during the

10 inspection process in a hazardous environment, we optimised and fabricated a magnetic sensor

11 that has a high resolution of $1 \mathrm{nT}$, which indicates a highly stable signal versus time and

12 temperature. The sensor is optimised based on our patented planar Hall effect (PHE) sensor [11]

13 with novel optimisation of thin film structure, sensor architecture and measurement configuration

14 to allow the measurement of high magnetic fields up to $50 \mathrm{mT}$. With these specifications, the

15 developed PHE sensor fulfils requirements to quantify the wall loss of a pipe using the flux

16 density method which is not achieved by conventional Hall sensors. The quantitative

17 measurements of thickness variation were illustrated on test pipes with a thickness change step as

18 small as $0.5 \mathrm{~mm}$.

\section{Theoretical description and finite element studies of the magnetic flux density method}

20 Fig. 1a shows a magnetic flux density measurement setup within a quadrant to highlight the

21 information of the magnetising coil, the test pipe and the air gap between them. The magnetic

22 flux in this air gap is the object of the investigation. 
1 To describe the physical principle of the pipe wall loss inspection based on magnetic flux density

2 method, we employed Ohm's law for magnetic circuits.

$$
F=\Phi R
$$

3 Where $F$ is the magnetomotive force (MMF) across a magnetic element, $\Phi$ is the magnetic flux $4 \quad$ through the magnetic element and $R$ is the magnetic reluctance of the element.

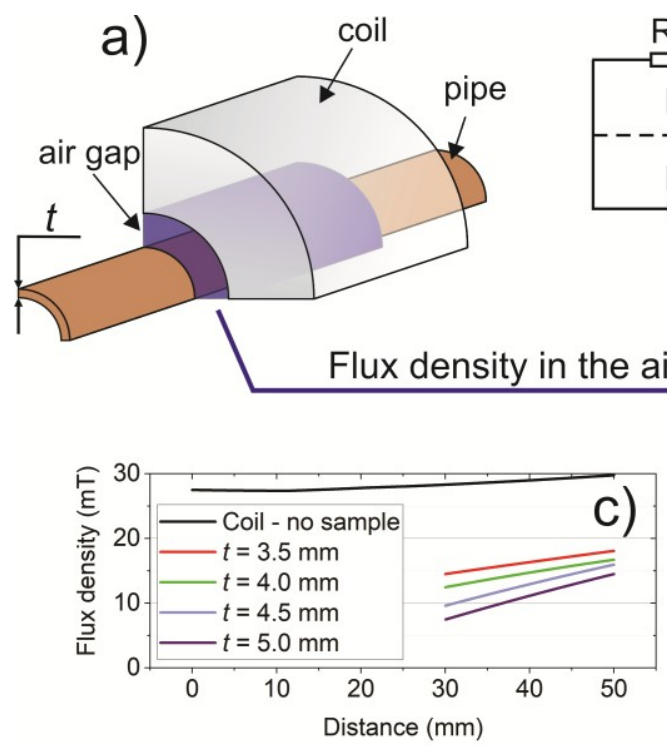

b)

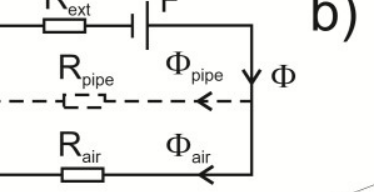

d)

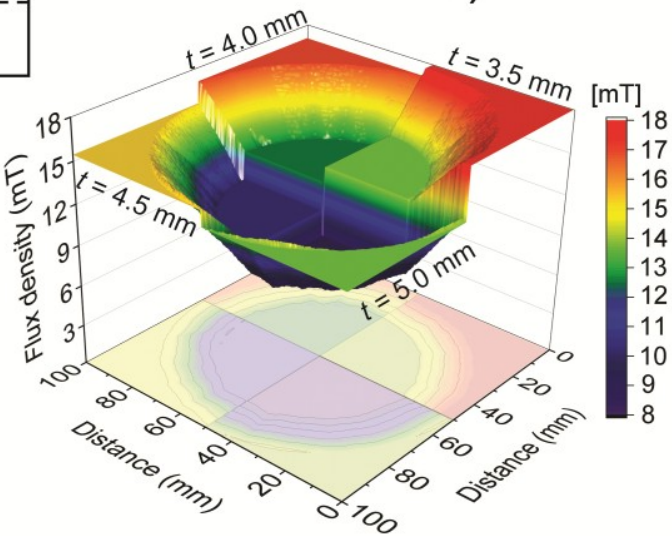

Fig. 1. (a) Schematic of the thickness monitoring based on the magnetic flux density method. (b) Equivalent magnetic circuits of the method. (c) The simulated flux density as a function of radial distance for four different thicknesses measured at $30 \mathrm{mT}$. (d) Four quadrants' colour plots of the cross section magnetic flux density in the air gap for four thicknesses of pipe wall $t=3.5,4.0,4.5$ and $5.0 \mathrm{~mm}$.

In Fig. 1b, $F$ denotes the MMF, which refers to the magnetic source in the term of the ampereturns (it is proportional to the applied magnetic field); and $R_{\text {ext }}, R_{\text {air }}$ and $R_{\text {pipe }}$ denote the magnetic reluctances in the space outside the magnetising coil, inside the magnetising coil and in the test

12 pipe, respectively. $\Phi, \Phi_{\text {air }}$ and $\Phi_{\text {pipe }}$ denote the total magnetic flux produced by $F$, the magnetic 13 flux through the air gap in the coil and through the pipe, respectively. 
1 According to Kirchhoff's law of magnetic circuit,

$$
\begin{aligned}
& \Phi_{\text {air }}=\frac{F-\Phi R_{\text {ext }}}{R_{\text {air }}} \\
& \Phi=\frac{F}{R_{\text {ext }}+R_{\text {equi }}}
\end{aligned}
$$

2 where $R_{\text {equi }}$ is the equivalent reluctance defined as

$$
\frac{1}{R_{\text {equi }}}=\frac{1}{R_{\text {air }}}+\frac{1}{R_{\text {pipe }}}
$$

3 where $R_{\text {pipe }}=\frac{1}{\mu} \frac{l}{A}$, with $\mu, l$ and $A$ are the magnetic permeability, the length and the cross-

4 sectional area of the test pipe, respectively.

5 With $\Phi$ in Equation 2 substituted with Equation 3, $\Phi_{\text {air }}$ can be rewritten as

$$
\Phi_{\text {air }}=\frac{F\left(1-\frac{R_{\text {ext }}}{R_{\text {ext }}+R_{\text {equi }}}\right)}{R_{\text {air }}}
$$

6 In Equation 4, the equivalent reluctance $\left(R_{\text {equi }}\right)$ decreases with decreasing $R_{\text {pipe }}$. As a result, $\Phi_{\text {air }}$

7 (Equation 5) decreases with decreasing $R_{\text {pipe }}$ (increasing pipe thickness). Therefore, the magnetic

8 flux in the air gap decreases with the increment of the thickness of a test pipe. Also, in Equation

95 , we expect that the wall loss signal increases with the applied field (proportional to $F$ ).

10 To estimate the change of magnetic flux as a function of thickness reduction quantitatively, we

11 employed finite element method (FEM) in ANSYS Maxwell software to simulate the change in

12 the magnetic flux density at an off-axis point when a pipe with a given diameter and a given

13 thickness is placed in the coil. In the FEM calculation, the magnetic field is described by 14 defining the geometric properties, material properties, currents and boundary conditions. In the 15 simulation, the magnetic field in a conducting domain satisfies the following system of 16 equations: 
Accepted MS: MEASUREMENT, Journal of the International Measurement Confederation

$$
\Delta \vec{B}=0 ; \Delta \vec{H}=\vec{J} ; \vec{B}=\mu_{0}(\vec{H}+\vec{M}) ; \overrightarrow{M_{\mathrm{t}}}=\overrightarrow{M_{\mathrm{t}}}(\vec{H})
$$

1 where $H$ is the magnetic field intensity, $J$ is the total current density, $B$ is the magnetic induction

2 quantity, $M_{t}$ is the temporary magnetisation of the material and $\mu_{0}$ is the vacuum permeability.

3 Maxwell's equations are solved with set of provided boundary conditions and boundary

4 constraints. The nodal values of the magnetic vector potential are considered as primary 5 unknowns and their derivatives i.e., flux density values are the secondary unknowns. In order to 6 obtain flux density values, the Sparse solver is employed. In our simulation, the steel 1010 is 7 chosen for the test pipe with the $B-H$ curve being taken from the library of the ANSYS Maxwell 8 software. The simulation was conducted with the system having the same parameters as those 9 used for the experimental setup, specifically, the magnetising coil has a length of $150 \mathrm{~mm}$, an 10 inner diameter of $100 \mathrm{~mm}$ and an outer diameter of $200 \mathrm{~mm}$. The length of the pipes is $700 \mathrm{~mm}$ 11 and their thicknesses are 3.5, 4.0, 4.5 and $5.0 \mathrm{~mm}$. The applied magnetic field is $30 \mathrm{mT}$.

12 The simulated results shown in Fig. 1c illustrate four plots of the magnetic field versus the radial 13 distance of four pipes with thicknesses of 3.5, 4.0, 4.5 and $5.0 \mathrm{~mm}$. The distribution of the 14 magnetic field inside the magnetising coil without a sample is also plotted (non-sample case). 15 The sensor lift-off effect can be seen clearly for the four investigated pipe's thicknesses by an increase of the flux density as a function of radial position from the outer surface of the pipe to 17 the inner surface of the magnetising coil. The split among four plots, i.e. the wall loss signal, is 18 higher at the outer surface of the pipe than that at the inner surface of the coil. Quantitatively, 5 $19 \mathrm{~mm}$ wall thickness reduction ( $10 \%$ wall loss) induces a decrease of flux density from $2.3 \mathrm{mT}$ to $201.2 \mathrm{mT}$ with radial position moving from the outer surface of pipe to the inner surface of 21 magnetising coil. 
Importantly, at any radial distance, the wall loss signal increases proportionally with the amount

2 of the wall loss. Therefore, we could locate the sensor at an appropriate radial position in order to

3 obtain, on the one hand good signal and on the other hand minimising MFL effect. For a visual

4 recognition of the change in the magnetic flux density in the air gap between the test pipe and the

5 magnetising coil due to the different thickness of pipes, we show in Fig. 1d the simulated results

6 of magnetic flux through 3D colour plots of the cross section with an identical colour scale. In

7 this figure, we can see a change in the magnetic flux density via the change of the colour and of

8 the magnetic flux density magnitude of four quadrants.

9 In short, the simulation exhibits the capability of the flux density method in wall loss inspection.

10 The change in magnetic flux density depends on the radial position, decreasing from the outer 11 radius of the test pipe to the inner radius of the magnetising coil.

\section{3. System design}

13 3.1. Optimisation and fabrication of ultrasensitive and low-noise magnetic sensor for high magnetic field measurement

15 The design and optimisation of a sensor in this work are derived from our previous development of PHE sensors with approved high sensitivity in the detection of a small quantity of magnetic

17 materials down to $10^{-14}$ emu. This sensitivity is 10,000 times higher than that of a 18 superconducting quantum interference device $[12,13]$, with a very high thermal stability of at 19 least 50-fold higher than that of a conventional Hall effect sensor [14]. These excellent 20 achievements are due to the nature of the sensor's architecture with a diagonal arrangement of 21 biasing current and sensing voltage directions $[15,16]$. With its high sensitivity, the PHE sensor 
1 exhibits its advantages in the detection of small and shallow defects in pipe walls on the basis of

2 magnetic flux leakage measurement [17].

3 To design a sensor working at high magnetic fields, we consider the optimisation of the sensor

4 from the fundamental background of the PHE sensor. The PHE voltage $\left(V_{\mathrm{PHE}}\right)$ varies with the

5 magnetic field due to the rotation of magnetisation from its easy axis towards the applied field

6 direction, as shown in Equation 7 [14]

$$
V_{\mathrm{PHE}}=V_{\mathrm{offset}}+V_{0} \cos \theta \sin \theta
$$

7 where $\theta$ is the magnetising angle; $V_{0}$ is a planar Hall voltage coefficient dependence bias current, 8 geometrical dimension and anisotropic resistivity of the sensor junction; and $V_{\text {offset }}$ is the offset 9 voltage.

10 The magnetising angle $\theta$ depends on the physical parameters of the sensor's stack expressed in 11 the magnetic energy $(E)$ equation with the contributions of anisotropic energy, exchange bias 12 energy and Zeeman energy as expressed in the first, second and third terms, respectively. [18]

$$
E=K_{\mathrm{u}} \sin ^{2} \theta-H_{\mathrm{ex}} M_{\mathrm{s}} \cos (\beta-\theta)-H M_{\mathrm{s}} \sin (\alpha-\theta)
$$

13 where $K_{\mathrm{u}}$ is the anisotropic constant, $H_{\mathrm{ex}}$ is the exchange bias field, $M_{\mathrm{s}}$ is the saturated 14 magnetisation, $H$ is the external magnetic field, $\alpha$ is the angle between the directions of the 15 applied magnetic field and easy axis and $\beta$ is the angle between the directions of bias current and exchange bias field/easy axis.

17 At an applied field value $H$, the magnetisation vector rotates at an angle $\theta$ towards the field 18 direction. The value of magnetising angle $\theta$ can be calculated while considering its minimum 19 energy state $\frac{\partial E}{\partial \theta}=0$. The dependence of $H$ on $\theta$ can be expressed by 


$$
H=\frac{K_{\mathrm{u}} 2 \sin \theta \cos \theta-H_{\mathrm{ex}} M_{\mathrm{s}} \sin (\beta-\theta)}{M_{\mathrm{s}} \sin (\alpha-\theta)}
$$

1 To maximise the sensitivity of a PHE sensor within a defined area ( $V_{0}$ in Equation 7$)$, we design

2 a meander-type sensor junction connected in the Wheatstone bridge configuration (Fig. 2a). The sensor junction was fabricated by photolithography technique with the optimised lift-off protocol

4 [19]. The sensor stack was deposited using a DC sputtering system. The electrodes were made of $\mathrm{Au}$ of $100 \mathrm{~nm}$. For good compatibility with wafer-level chip scale packaging, the size of the electrodes and pitch between them was 200 and $400 \mu \mathrm{m}$, respectively.
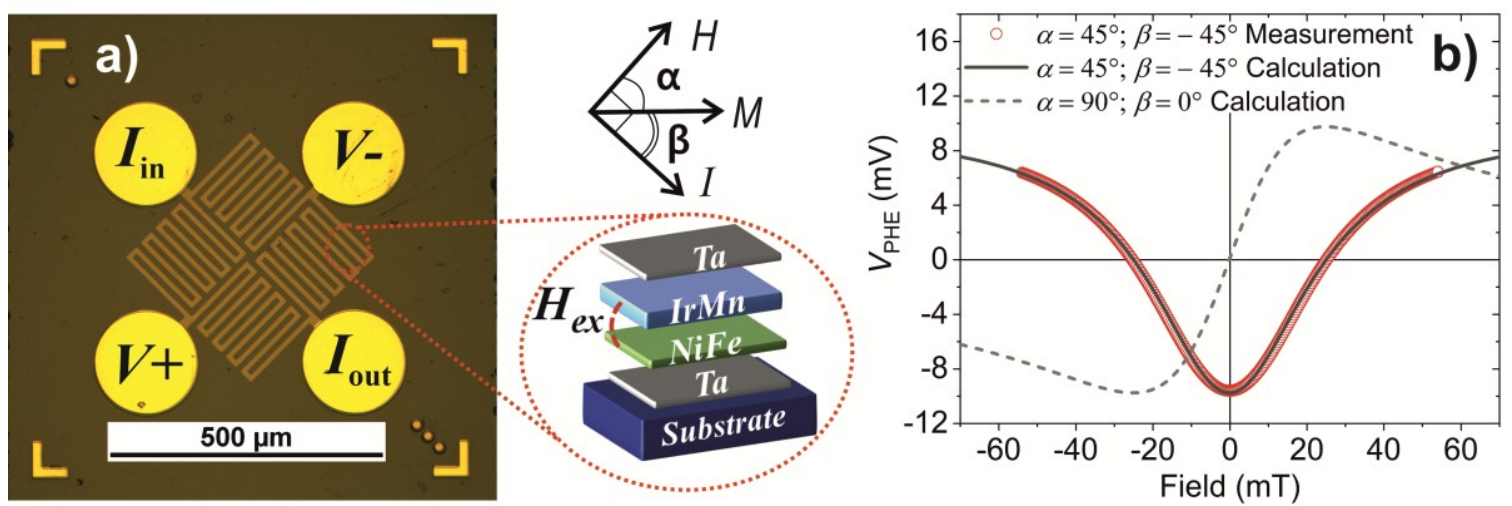

Fig. 2. (a) Image of a PHE sensor. The electrodes for biasing current and measured voltage are denoted on the corresponding pads. The external magnetic field and biasing current are applied in directions away from easy magnetisation axis angles $\alpha$ and $\beta$, respectively. (b) Calculations of PHE voltage profiles for two cases ( $\alpha=90^{\circ}$ and $\beta$ $\left.=0^{\circ}\right)$ and $\left(\alpha=45^{\circ}\right.$ and $\left.\beta=-45^{\circ}\right)$. For measuring a wide field range, the best measurement configuration is $\alpha=45^{\circ}$ and $\beta=-45^{\circ}$. Therefore, the sensor was optimised and fabricated in this configuration. The measurement voltage profile of the optimised sensor is presented together in the figure for evidence.

For high field measurement, a high exchange bias field $\left(H_{\mathrm{ex}}\right.$ in Equation 9$)$ is required.

14 Therefore, we optimised an exchange bias structure with a small thickness of the ferromagnetic layer Ta/Py/IrMn/Ta $(5 / 4 / 10 / 5 \mathrm{~nm})$ for sensor stack, where Py is Permalloy 80. With our fabrication condition, the exchange bias field of the sensor stack is about $25 \mathrm{mT}$. 
In a conventional measurement configuration of a planar Hall sensor, the applied field is

2 perpendicular to the easy axis of magnetisation vector $\left(\alpha=90^{\circ}\right)$ and the current is biasing in the

3 same direction of the magnetisation $\left(\beta=0^{\circ}\right)$. In this case, the $V(H)$ profile changes linearly in

4 small fields within the interval of $\pm\left|H_{\text {ex }}\right|$, as shown by the dashed curve plotted in Fig. 2b. With

5 this measurement configuration, the sensor is not sufficient to be used as a detector for magnetic

6 flux density measurement in this work, which requires a high magnetic field measurement.

7 To increase the measured field range for the PHE sensor, we introduce a new measurement

8 configuration. By analysing the $V_{\mathrm{PHE}}(H)$ profile from our modelling reported earlier [18] given in

9 Equations (7) and (9), we find that the measurement field of a sensor can be extended to a higher

10 field value than $H_{\mathrm{ex}}$ when angles $\alpha$ and $\beta$ are $45^{\circ}$ and $-45^{\circ}$, respectively. Therefore, in the

11 fabrication process, we arrange the easy magnetisation axis, the biasing current direction and the

12 output voltage direction following these theoretical conditions. The measurement and calculation

13 voltage profiles of the sensor working in the new configuration $\left(\alpha=45^{\circ}, \beta=-45^{\circ}\right)$ are illustrated

14 in the solid line and scatter symbol curves in Fig. $2 b$.

15 With this new optimisation, the sensor is able to detect magnetic fields in the range from 0 to $1650 \mathrm{mT}$ with high sensitivity. Therefore, it fulfils the requirement for use in the magnetic flux 17 density measurement system in this work.

\subsection{Low-noise magnetic field measurement system}

A schematic and an image of the PHE magnetometer are illustrated in Fig. 3. The output voltage

20 of the PHE sensor is connected to a two-stage amplifier electronic system (Fig. 3a). The first

21 amplifier (Amp 1) amplifies the output voltage of the PHE sensor to the full voltage range of the

22 electronic system, then it is converted to a digital voltage by a 16-bit analog-to-digital converter 
1 (ADC) to send the data to a computer through the universal asynchronous receiver transmitter

2 connection. The second amplifier (Amp 2) is for calibrating the measurement at zero field or any

3 measurement field background of interest. When the set zero function is activated, the

4 microcontroller (MC) measures the output voltage of the amplifier 1 , known as $V_{1}$. Then, the MC

5 manages the digital-to-analog converter (DAC) to send the same voltage $V_{1}$ to the inverted input

6 of amplifier 2. Consequently, the differential voltage at the output of the amplifier 2 will be zero.

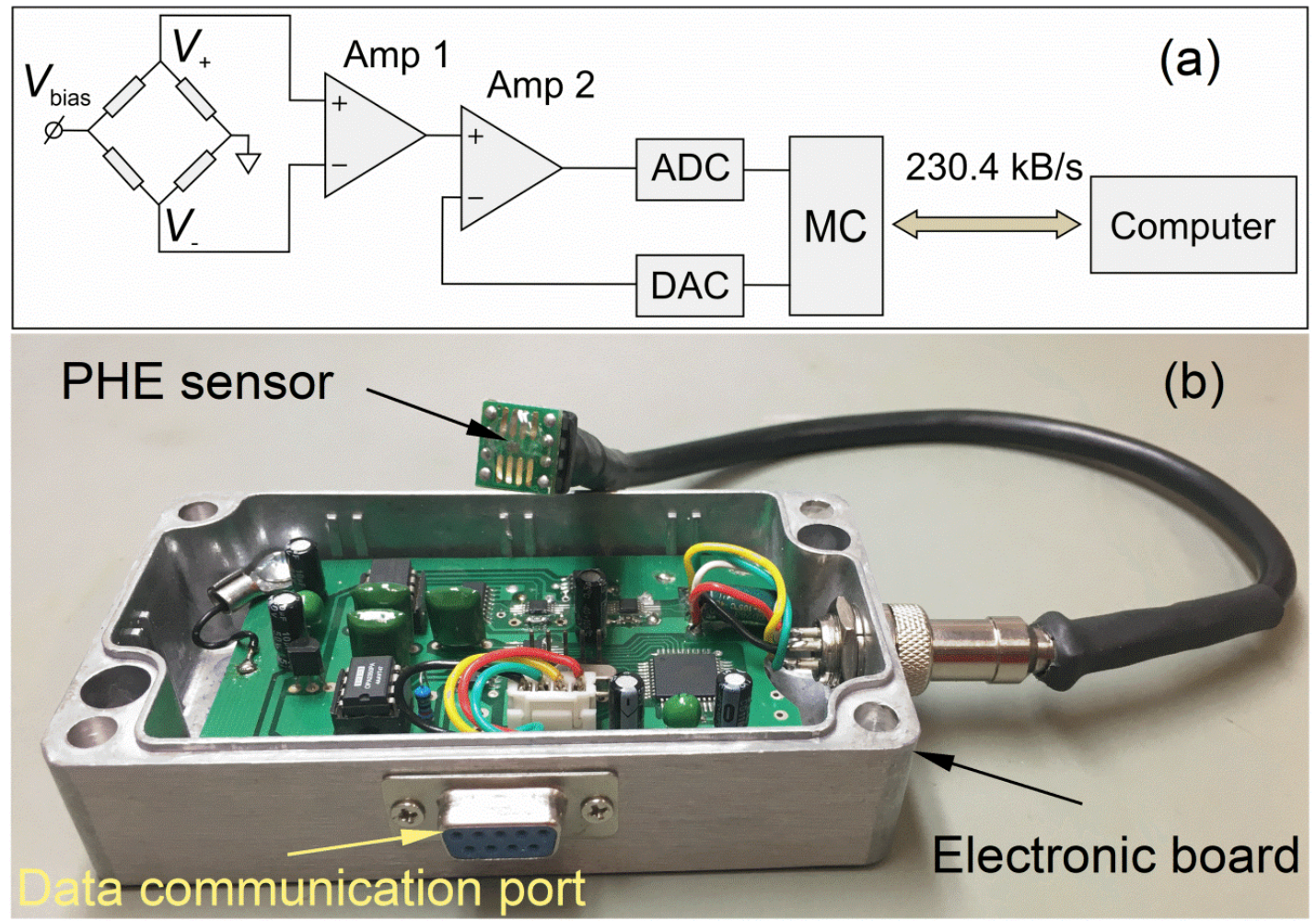

7 Fig. 3. (a) Schematic of the electronic circuit for the optimised PHE sensor with auto zero-field calibration function. (b)

8 Image of the PHE micromagnetometer, including the optimised PHE sensor, low-noise electronic circuit and data communication shielded port for high-speed data transmission to a computer at a baud rate of $230.4 \mathrm{k} \mathrm{Mb} / \mathrm{s}$.

The PHE sensor is connected to a gold-plated printed circuit board by using a wire bonding machine (WestBond 7700D). Then, the sensing area is protected by UV epoxy for mechanical 
1 protection. This magnetic sensing head is connected to the electronic board through a shielded

2 cable so that the sensor probe is able to access the measurement point easily (Fig. 3b).

3 The data is transmitted to the computer at a high baud rate of $230.4 \mathrm{kB} / \mathrm{s}$ through a data

4 communication port. This high transmission rate will be an advantage for fast thickness

5 monitoring with high resolution. A software is designed to collect data and convert the voltage

6 values into actual magnetic field values. Precise calibration was conducted prior to the thickness

7 monitoring by using the voltage versus field fitting function discussed in the previous section.

8 With these developments, the PHE magnetometer system has resolutions of $0.01,0.1$ and $1 \mu \mathrm{T}$

9 corresponding to the measurement ranges of $\pm 0.35, \pm 3.5$ and $\pm 35 \mathrm{mT}$, respectively. These

10 resolutions are solely limited by the accuracy of the 16-bit ADC and electronic components but

11 not caused by the sensor's resolution itself. Interestingly, with the high thermal stability and

12 high-resolution properties of the optimised PHE sensor, we achieved a stable field measurement

13 for several hours without any calibration process.

\subsection{Magnetic flux density measurement system}

15 In our experiment, the magnetiser is Helmholtz coils (Model: BH156-HPHF-B Ferronato ${ }^{\mathrm{TM}}$, 16 Spain). The inner diameter is $100 \mathrm{~mm}$, the outer diameter is $200 \mathrm{~mm}$, the length of the coils is

$17150 \mathrm{~mm}$ and the field generated by the coils is $5 \mathrm{mT} / \mathrm{A}$ with a maximum field of $50 \mathrm{mT}$. The 18 current is supplied by a bipolar power supply $400 \mathrm{~W}$ (BOP 36-12, Kepco Inc.) with a resolution 19 of $0.18 \mathrm{~mA}$. Therefore, the field resolution of the Helmholtz coils is $9 \mathrm{nT}$.

20 The magnetic fields were altered by varying the applied current to the Helmholtz coils to 10,15 , $2120,25,30$ and $35 \mathrm{mT}$. The test pipes can travel through the magnetiser by moving the pipes on a 
conveyor system (Fig. 4a). A plastic supporting pipe is used to keep the movement of the

2 ferromagnetic pipe (test sample) stable.

3 Magnetic field variation in these measurements is probed by the optimised PHE magnetometer,

4 which is located at the half distance between the outer surface of the pipe and the inner surface of

5 the magnetising coils.

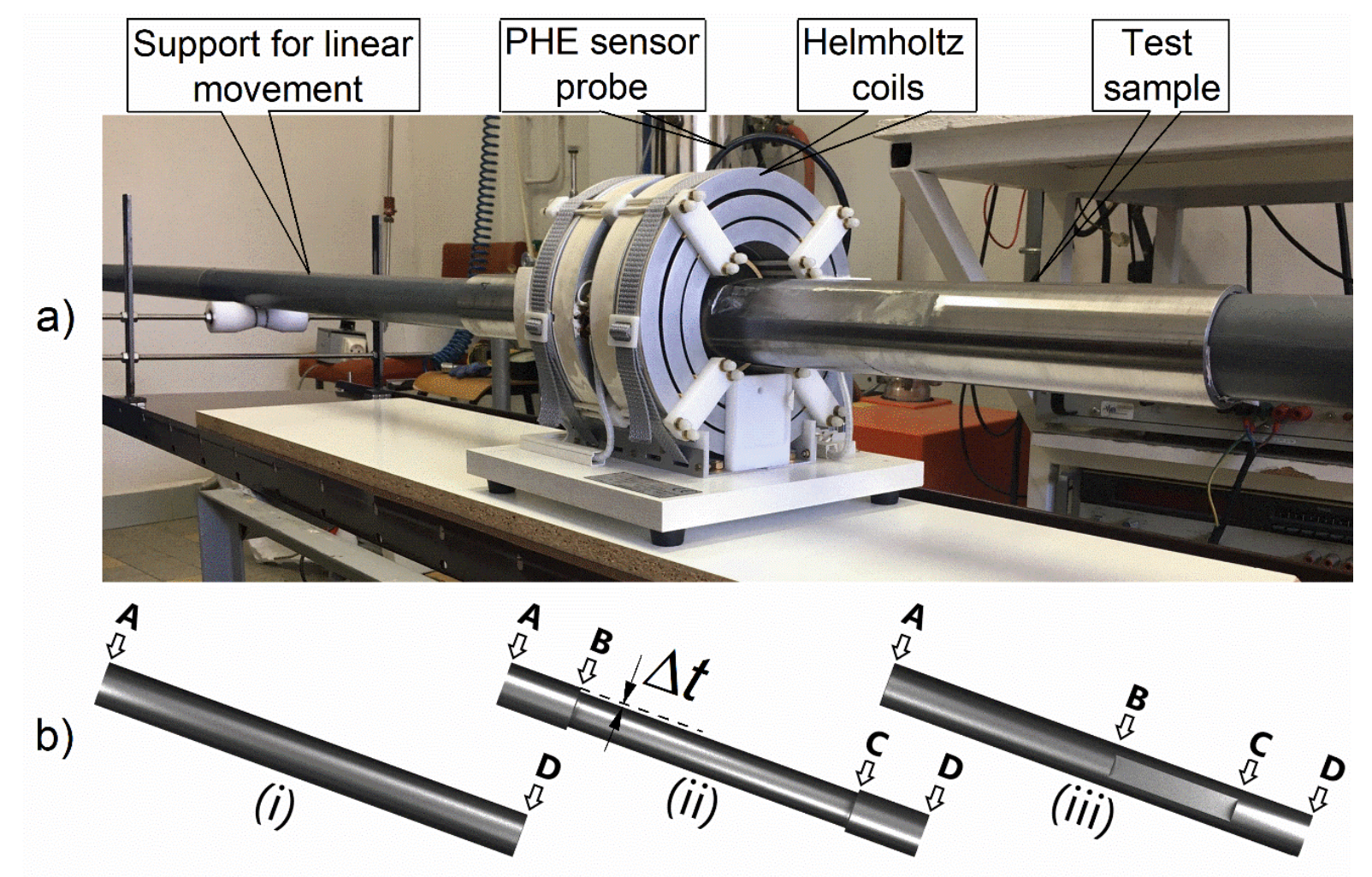

Fig. 4. (a) Experimental setup of the thickness monitoring system. (b) test pipes correspond to three cases: (i) the reference pipe with non-reduction of pipe wall, (ii) uniform wall thickness reduction of $\Delta t=0.5,1$ and $1.5 \mathrm{~mm}$ and (iii) artificial eccentric wall reduction.

\subsection{Test sample}

Test samples are five pipes made of the same material with an identical length of $700 \mathrm{~mm}$, an 
outer diameter of $60 \mathrm{~mm}$ and a thickness of $5 \mathrm{~mm}$ (Fig. 4b). The reference pipe does not contain

2 any loss $(i)$. Pipes 2, 3 and 4 contain a part with a length of $500 \mathrm{~mm}$ whose wall thickness is

3 reduced by $\Delta t=0.5,1.0$ and $1.5 \mathrm{~mm}$, respectively $(i i)$. The locations of the ends of the pipes and

4 the edges of wall loss are denoted by the letters A, B, C and D. Pipe 5 is an eccentric wall loss

5 pipe in which a part of the pipe is artificially cut $3 \mathrm{~mm}$ down and the length of the wall loss path

6 is $200 \mathrm{~mm}$ (iii). Because the change of flux density is independent of the wall loss position along

7 the pipe's circumference whether it is locating at the inner or outer surface, artificial wall losses

8 in this experiment set are made on the outer surface of the investigated pipes. However, the

9 quantification results are generalised for any location of the wall loss.

\section{Results and discussion}

\subsection{Measurement of eccentric wall loss using one measurement point}

12 In wall thickness monitoring based on magnetic flux density, two different conclusions are given

13 with regard to the ability of the method to detect wall loss. William Walters et al. [9] reported

14 that when a pipe with a localised wall reduction is inspected by using the magnetic flux density 15 method, the distribution of the flux lines is defined locally along the geometric shape of the anomaly. On the basis of this finding, they arranged their measurement setup, which consisted of

17 four sensors located in four quadrants around the test pipe. In contrast, W.D. Averitt [20] 18 reported that as the thickness on one side of the pipe changes, the flux density changes reason, only one sensor may be needed for wall loss monitoring. 

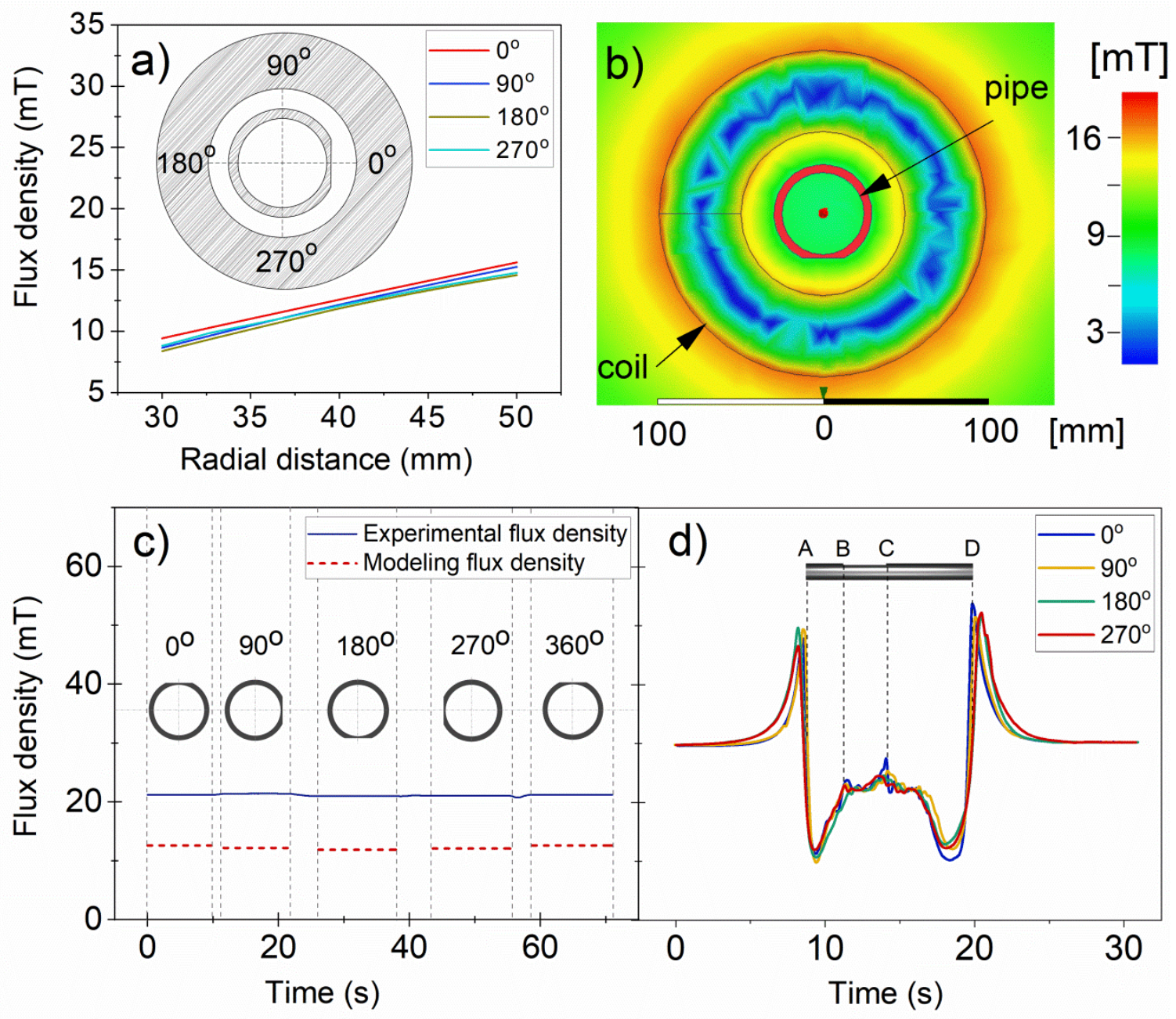

1 Fig. 5. (a) Simulation results of magnetic flux densities of an eccentric wall loss pipe at four quadrants $0^{\circ}, 90^{\circ}, 180^{\circ}$ and $270^{\circ}$, illustrated in the inset. (b) Colour plot of magnetic flux distribution around an eccentric wall loss pipe. (c) The measured and simulated results of flux densities around the eccentric wall loss pipe. (d) Experimental results of

4 the magnetic flux density along an eccentric wall loss pipe at four angular positions of $0^{\circ}, 90^{\circ}, 180^{\circ}$ and $270^{\circ}$ at the 5 applied field of $30 \mathrm{mT}$.

6 To determine the required number of sensors in our measurement system, we conducted a 7 simulation for an eccentric wall loss pipe with a thickness of $5 \mathrm{~mm}$ and an artificial wall loss of 3 $8 \mathrm{~mm}$. Fig. 5a shows the simulated magnetic flux densities at four angular positions (inset) versus 9 radial distance when the applied field is $30 \mathrm{mT}$. All the four curves are not split clearly and the 
1 flux density around the eccentric wall loss pipe changes only slightly. The 2D colour plot of the

2 cross section magnetic flux density around the test pipe is shown in Fig. 5b. The colour

3 distribution around the test pipe is almost symmetric.

4 In Fig. 5c, the flux density around the eccentric wall loss pipe at the applied field of $30 \mathrm{mT}$ are

5 plotted together with the calculated results for the quadratic rotary scan (the pipe rotates in 6 quadratic steps about the pipe axis) as solid and dashed lines, respectively. The calculated and

7 measured magnetic flux densities at four quadratic positions are evidently quite similar.

8 Therefore, through simulation and experimental verification, we agree with the work reported by

9 W.D. Averitt that using one sensor element is sufficient to monitor the wall thickness loss of

10 both eccentric and symmetric wall loss types.

11 To verify this conclusion, we performed measurements using one probing point to monitor the

12 eccentric wall loss pipe by recording the flux density along the length of the test sample under an 13 applied field of $30 \mathrm{mT}$, as shown in Fig. 5d. In these measurements, the test pipe with eccentric 14 wall loss aligned with one sensor element at four angular positions of the test pipe of $0^{\circ}, 90^{\circ}$, $15180^{\circ}$ and $270^{\circ}$. We observed in all four angular positions an identical increment of the magnetic 16 field with an average value of $1.3 \mathrm{mT}$ for the $8 \%$ eccentric wall loss pipe. The percentage of wall 17 loss is calculated in volume from the geometric dimensions of the pipe and the eccentric wall 18 loss. The MFL effect caused by the edges of the thin region also contributed to this magnetic 19 field increment. We will discuss this observation further in Fig. 6. More importantly, 20 experimental verification shows that the similarity of the four curves measured at four angular 21 positions of the eccentric wall loss pipe indicates that the detection of an eccentric wall loss 22 requires only one measurement point. This verification strengthens our statement in the 
introduction section that the flux density is identical along the circumference of a pipe regardless

2 the positions of metal losses.

\section{$3 \quad 4.2$ Quantification of the thickness reduction of pipe's wall}

4 In the following, we perform a measurement set to quantify the change in the magnetic flux

5 density versus reduction of wall thickness. Prior to these quantification measurements, we

6 characterise a reference pipe with no thickness reduction (pipe (i) in Fig. 4b) at several applied

7 fields to establish background signals.

8 Fig. 6 shows three selected results for the reference pipe measured at applied fields of 10, 25 and

$935 \mathrm{mT}$. Firstly, in the flux density versus time profiles, we observe two straight lines that

10 correspond to the non-sample periods. The high stable signal in these two parts reveals the good

11 quality of the optimised PHE magnetic measurement system. Secondly, the moments that

12 correspond to the starting and ending pipe scans are denoted by the letters A and D on the plots,

13 respectively. At around these positions, we clearly observe two strong peaks that are no doubt

14 attributed to the edge effect, which is the contribution of the magnetic flux lines coming in and

15 out from the two ends of the pipe. At the starting and ending points, this contribution changes

16 abruptly from positive to negative. However, in the actual monitoring process, this effect has a

17 small contribution when the pipe's ends move far away from the coil. In our experiment, the test

18 pipes are rather short; therefore, the edge effect may exist in all measurements, leading to a non-

19 flat base line. Lastly, this background signal will be subtracted in all the thickness measurements

20 so it does not affect the quantitative results. 
1 In fact, the inspected pipes are much longer than the test pipes in our experiment. Therefore, the

2 fluctuation of the flux density near the edge will not contribute considerably to the actual flux

3 density measurements. For the drill pipes in particular, the inspection procedure will bypass a 4 tool joint with a length of about $300 \mathrm{~mm}$.

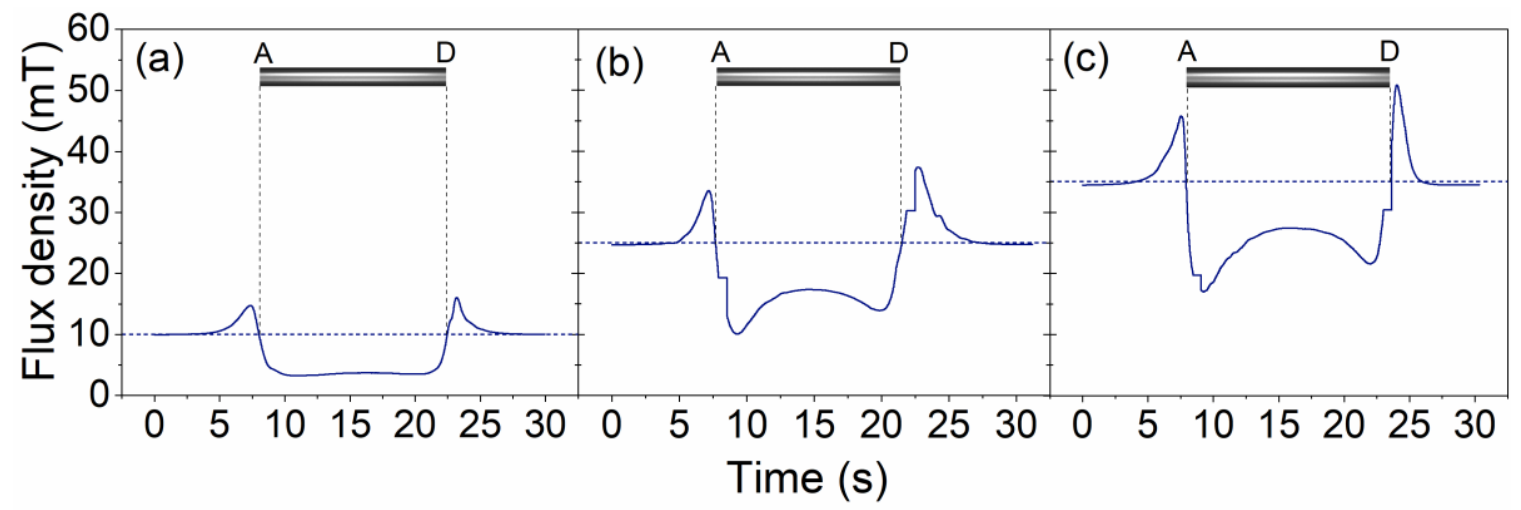

5 Fig. 6. Plots of flux densities measured along the reference pipe at applied fields of $10 \mathrm{mT}(\mathrm{a}), 25 \mathrm{mT}$ (b) and $35 \mathrm{mT}$ 6 (c). The applied field values are represented by horizontal dotted lines.

7 Fig. 7 shows results measured at three applied fields of 10, 25 and $35 \mathrm{mT}$ for pipes 2, 3 and 4 . In 8 these flux density measurements, the moments when the edges of the thin wall pass by the sensor 9 are denoted on the plots by the letters B and C. These points are identified by the anomalies that appear on the base line due to the MFL phenomenon. In these measurements, the MFL effect is

11 pronounced because of the small proximity of the sensor to the surface of the test pipe as a result

12 of the small internal diameter of the magnetising coil. The MFL also causes the signal in the 13 region that corresponds to the thin part to fluctuate; this fluctuation is a result of the rough 14 surface and/or a local variation of the magnetic permeability due to the high temperature during 15 the mechanical engineering process of manufacturing the artificial wall reduction samples. 


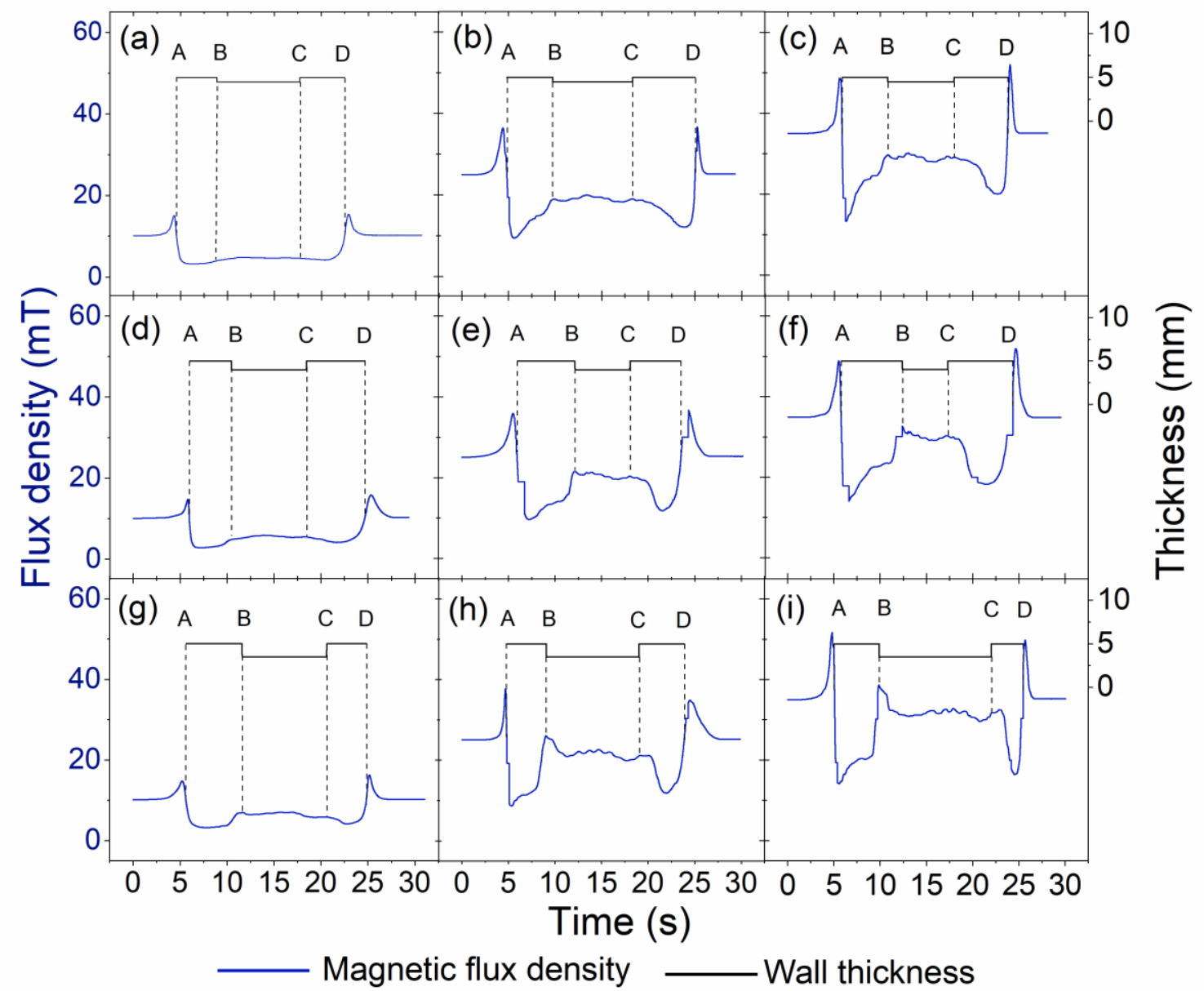

1 Fig. 7. Flux density curves measured at applied fields of 10,25 and $35 \mathrm{mT}$ for pipes 2,3 and 4 with wall thickness reduction of $0.5 \mathrm{~mm}(\mathrm{a}-\mathrm{c}), 1.0 \mathrm{~mm}(\mathrm{~d}-\mathrm{f})$ and $1.5 \mathrm{~mm}(\mathrm{~g}-\mathrm{i})$.

3 However, the most important observation in this measurement set is the increment in the

4 magnetic flux density in the air caused by the wall thickness reduction $(\Delta t)$. The change in the 5 magnetic flux density versus the wall thickness reduction (signified by $\Delta B$ ) is the difference

6 between the magnetic fields of reference pipes and wall loss pipes. The field values were taken

7 in the middle of the thin-wall part of the wall loss pipes and in the middle of the reference pipe.

8 Therefore, the fluctuating signals due to the MFL effect at points B and C are not integrated in 9 the deduction values of $\Delta B$. The results are plotted in Fig. 8. 


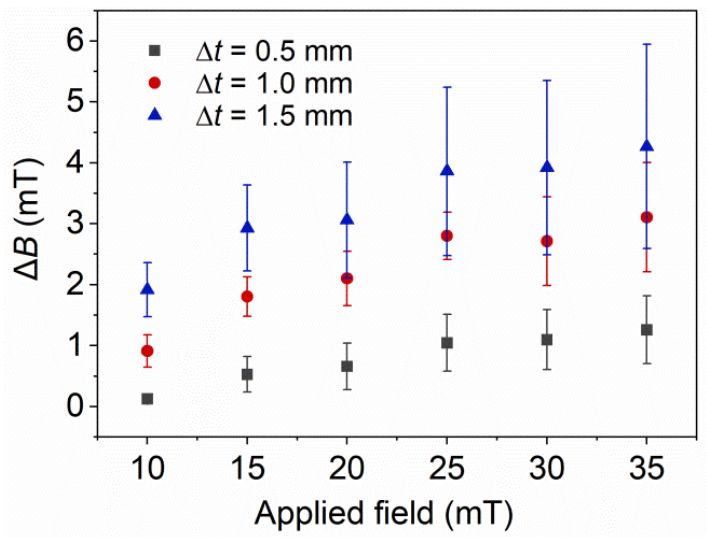

1 Fig. 8. Experimental results of the flux density change $(\Delta B)$ for wall thickness losses $\Delta t=0.5,1.0$ and $1.5 \mathrm{~mm}$ at 2 several measured fields.

3 From these findings, we highlight the following remarks:

4 (1) The flux density change with wall loss $(\Delta B)$ increased with the applied magnetic field for all the investigated thickness losses. This observation can be understood from the analytical approach in Equation 5. As a result, wall loss can be detected more easily at a high applied field than at a low applied field.

(2) The change in the magnetic flux density caused by wall thickness reduction is observed clearly and the wall loss signal $\Delta B$ increases linearly with the cross-sectional area of wall loss. Therefore, we can correlate wall loss with $\Delta B$ as a monitoring function of the thickness loss, thereby allowing the thickness reduction of a pipe to be monitored directly.

12 (3) At the measurement condition, the wall loss signal $\Delta B$ for $10 \%$ wall loss of a pipe is about $131.4 \mathrm{mT}$. This value is rather low compared to the applied fields of $30 \mathrm{mT}$. As a result of this condition, a PHE sensor that has a high sensitivity and signal stability with time and 
Accepted MS: MEASUREMENT, Journal of the International Measurement Confederation

temperature needs to be developed to facilitate the quantitative measurement of small thickness loss.

\subsection{Factors and limitation of the method}

Arcording to the "Guide to the Expression of Uncertainty of Measurement" (GUM). ISO / IEC Guide 98-1: 2009, Uncertainty of measurement - Part 1: Introduction to expressing uncertainty of measurement, IDT" [21], quantification data in this work is clasified as uncertainty measurements. Therefore, the quantification data discussed in Fig. 6, Fig. 7 and Fig. 8 are represented for one study case. In general, there are several major factors affecting the integrity of the wall monitoring process.

The sensor lift-off effect is presented in section 2 that the flux density background signal is decreased and the signal change versus wall loss is increased from the outer surface of the pipe to inner surface of magnetising coil. In this context, the quantification data is accurate only when the sensor is located at the same lift-off distance in all measurements.

The wobble effect causing by the off-centre coaxial alignment of the pipe to the magnetising coil contributes significantly to the inaccuracy of the measurement. In this early stage of the development, the wall thickness monitoring system is utilised in combination with a hydraulic conveyor system to ensure the pipe being coaxial to the coil and so to minimise the wobble effect. Further development on novel configurations of the system and optimising methods for the data acquisition should be done. In this case, system designs, measurement techniques and algorithm methods developed to minimise the wobble effect in eddy current testing [22, 23] should be referenced. 
1 There exists edge effect and MFL effect appeared in all investigated samples as well as in the

2 real inspection pipes, so that the edge effect needs to be removed from the thickness reduction

3 measurement. To avoid the influence of the edge effect, the procedure should bypass the two

4 ends of pipes. The contribution of the MFL signal does not cause a shift of the signal but rather

5 cause the abrupt anomalies in the base signal, it is can be filtered out easily in the thickness

6 monitoring. In another sense, these anomalies are also useful contributed to identify defects of a

7 pipe.

8 Measurement the thickness change of unknown thickness and unknown permeability pipe is

9 important for validation of the flux density method. In our experiment, measurement of the wall

10 loss in an unknown thickness and unknown permeability pipe can be done by correlation with a

11 calibration sample of the same material. The most difficult problem to solve is the local variation

12 of the magnetic permeability in a pipe affected flux density signal. This phenomenon causes a

13 fluctuation of the measuring signal without the change of wall thickness. To solve this issue,

14 combination of the flux density measurement with an imaging process should be considered.

15 Another factor affected largely the accuracy of the thickness quantification is the thermal drift of 16 the sensor system; the thermal drift is a result of poor thermal stability of the sensor. This is the 17 main motivation for us to employ the PHE sensor with high temperature stability. The sensor 18 was investigated to confirm its excellent performance in an environment from cryogenic 19 temperature up to $150{ }^{\circ} \mathrm{C}$. To minimise the thermal drift of magnetic flux density caused by 20 temperature increasing of the magnetising coil, a DC source using a constant current mode is 21 recommended.

22 Other constrains of the flux density method could be that the method is applicable only for the 
1 ferromagnetic pipes and best suit for the pipes with small, straight and disjointed characteristics.

2 To examine the thickness at two ends of a pipe, employment of compensation process to remove

3 background signal caused by the edge effect is required.

\section{5. Conclusions}

5 This work reports the ability of the magnetic flux density method to quantify the wall loss of a

6 pipe that undergoes gradual wall reduction. With the use of a precise magnetic sensor

7 measurement system, the proportion of wall loss signal has been found experimentally to be 1.4

$8 \mathrm{mT}$ per $10 \%$ loss of wall thickness. By conducting a feasibility study of the method in detection

9 of the wall loss in quadrants, we confirm that one sensor is sufficient to monitor eccentric wall 10 loss.

11 To achieve this quantification approach, a planar Hall sensor with ultra-high-sensitivity, high

12 thermal stability and stable background signal versus time for the detection of high magnetic

13 fields was developed. In addition to the sensor development, a customised electronic system with

14 high precision is realised to measure the magnetic flux density in the air within the magnetising 15 coil and the test pipe. The quantification of thickness loss is proven through an investigation of 16 the flux density variation on a series of pipes with a thickness loss that varies with a $0.5 \mathrm{~mm}$ step.

17 With these encouraging results in quantification of metal losses, independence of the loss 18 position whether the loss is located at the inner surface or at the outer surface of a pipe, the flux 19 density method is interesting to non destructive tests. The pilot system presented in this paper 20 represents a single study case, there are several factors which could affect significantly to the 21 quantification results. In other setups and environmental conditions, results will be achieved 22 differently, however, the integrity of the wall loss quantification by using the flux density 
method is ensured.

\section{Credit Authorship Contribution Statement}

3 H.Q. Pham, Q.H. Tran: Conceptualisation, Methodology, Validation, Writing -original draft, 4 Project administration, Supervision. T.K. Nguyen, Q.N. Pham: Investigation, Visualisation, 5 Writing -review \& editing. V.T. Nguyen: T.T. Truong, M.H. Vu: Investigation, Writing 6 review \& editing. V.S. Le, A. Bousseksou, S. Wane, F. Terki: Resources, Writing -review \& 7 editing, Funding acquisition, Supervision

\section{Declaration of Competing Interest}

9 The authors declare that they have no known competing financial interests or personal 10 relationships that could have appeared to influence the work reported in this paper.

\section{Acknowledgements}

12 This work was supported by PetroVietnam Group -PVN (project no. 13 14/ATBVMT/PVU/2020/KHCN), PetroVietnam University-PVU (project no. 406/QĐ-ĐHDK), 14 and eV-Technologies -(R\&D on the development of the high-sensitivity magnetometer system). 15 We acknowledge Prof. CheolGi Kim at DGIST, South Korea, for the valuable discussion and 16 support on the fabrication of the PHE sensor. 


\section{References}

[1] M. Hirao, H. Ogi, An SH-Wave EMAT technique for gas pipeline inspection, NDT\&E Int., 32 (1999) 127-132, https://doi.org/10.1016/S0963-8695(98)00062-0.

[2] R. Jafari-Shapoorabadi, A. Konrad and A.N. Sinclar, Improved finite element method for EMAT array design, IEEE Trans. Magn., 37 (2001) 2821-2823, https://doi.org/10.1109/20.951317.

[3] D. Kim, L. Udpa, Remote field eddy current testing for detection of stress corrosion cracks transmission pipelines, Mater. Lett., 58 (2004) 2102-2104, https://doi.org/10.1016/j.matlet.2004.01.006.

[4] W. Zhang, Y. Shi, Y. Li, Q. Luo, A study of quantifying thickness of ferromagnetic pipes based on remote field eddy current testing, Sensors 18 (2018) 2769, https://doi.org/10.3390/s18092769.

[5] R.K. Stanley, Magnetic methods for wall thickness measurement and flaw detection in ferromagnetic tubing and plate, Insight, 38 (1996) 51-55.

[6] R.K. Stanley, Simple explanation of the theory of the total magnetic flux method for the measurement of ferromagnetic cross sections, Mater. Eval., 53 (1995) 72-75.

[7] Y. Zhang, G. Yan, Detection of Gas Pipe Wall Thickness Based on Electromagnetic Flux Leakage, Russ. J. Nondestruct. Test., 43 (2007) 123-132, https://doi.org/10.1134/S1061830907020088.

[8] B. Wijerathna, F.H. Ghorbel and W. Marsh, Measurement of pipe wall thickness using magnetic flux leakage signal, US8134360B2 (2012).

[9] W. Walters, D. Steely, Using magnetic flux density to identify anomalies in pipe wall thickness, Scan Systems Corporation.

[10] S. Tumanski, Thin film magnetoresistive sensors, IoP Publishing (2001).

[11] F. Terki, A. Busseksou, Q.H. Tran, S. Kamara, C.G. Kim, K.W. Kim, P.H. Gandit, Micromagnetometry detection system and method for detecting signatures of magnetic materials, EP2872911B1 (2016). 
[12] Q.H. Tran, F. Terki, S. Kamara, K.W. Kim, C.G. Kim, Planar Hall ring sensor for ultralow magnetic moment sensing, J. Appl. Phys. 117 (2015) 154505, https://doi.org/10.1063/1.4918534.

[13] S. Kamara, Q.H. Tran, V. Davesne, F. Gautier, L. Salmon, K.W. Kim, C.G. Kim, A. Bousseksou, F. Terki, Magnetic susceptibility study of sub-pico-emu sample using a micromagnetometer: An investigation through bistable spin-crossover materials, Adv. Mater., 29 (2017) 1703073, https://doi.org/10.1002/adma.201703073.

[14] M. Mahfoud, Q.H. Tran, S. Wane, D.T. Ngo, E.H. Belarbi, A. Boukra, M. Kim, A. Elzwawy, C. Kim, G. Reiss, B. Dieny, Reduced thermal dependence of the sensitivity of a planar Hall sensor, Appl. Phys. Lett., 115 (2019) 072402, https://doi.org/10.1063/1.5110671.

[15] A Schuhl, F.N.V. Dau, J.R. Childress, Low-field magnetic sensors based on the planar Hall effect, Appl. Phys. Lett., 66 (1995) 2751-2753, https://doi.org/10.1063/1.113697.

[16] T.H. Jeon, J.H. Lee, A. Talantsev, C.G. Kim, Planar Hall resistance sensor with improved thermal stability, IEEE Magn. Lett., 10 (2019) 8106305, https://doi.org/10.1109/LMAG.2019.2943054

[17] H.Q. Pham, B.V. Tran, D.T. Doan, Q.N. Pham, K.W. Kim, C.G. Kim, F. Terki, Q.H. Tran, Highly sensitive planar Hall magnetoresistive sensor for magnetic flux leakage pipeline inspection, IEEE Trans. Magn. 54 (2018) 6201105, https://doi.org/10.1109/TMAG.2018.2816075.

[18] Q. H. Tran, H. Q. Pham. T. T. Nguyen, S. Oh, B. Bajaj, C. G. Kim, The contribution of the exchange biased field direction in multilayer thin films to planar Hall resistance, Phys. Stat. Sol. (b), 17 (2007) 4431-4434, https://doi.org/10.1002/pssb.200777309.

[19] Q. H. Tran, D. Y. Kim, B. P. Rao, C. G. Kim, Novel planar Hall sensor for biomedical diagnosing lab-on-a-chip, State of the Art in Biosensors - General aspects, (2013), https://doi.org/10.5772/52820.

[20] W.D. Averitt, Magnetic wall, The International Pipe Inspectors Association (IPIA) edition of Crosstalk, New Tech System (2004), http://www.newtechsystems.com/article1 [Retrieved Nov. 2020]. 
Accepted MS: MEASUREMENT, Journal of the International Measurement Confederation

[21] JCGM 100:2008. Evaluation of measurement data - Guide to the expression of uncertainty in measurement (GUM). Joint Committee for Guides in Metrology

[22] W. Yin, R Binns, S.J. Dickinson, C. Davis, A.J. Peyton, Analysis of the Lift-off Effect of Phase Spectra for Eddy Current Sensors, IMTC 2005 - Instrumentation and Measurement Technology Conference Ottawa, Canada, 17-19 May 2005.

[23] L. Shu, H. Songling, Z. Wei, and Y. Peng, Improved Immunity to lift-off effect in pulsed eddy current testing with two-stage differential probes, Russian Journal of Nondestructive Testing, 44 (2008) 138-144. 